\title{
Soft Computing Modelling of Urban Evolution: Tehran Metropolis
}

\author{
Mostafa Borhani*, Nima Ghasemloo \\ Shahid Beheshti University, Quran Miracle Research Institute, 1983963113, Evin, Tehran, I.R. (Iran) \\ Received 14 August 2018 | Accepted 15 February 2019 | Published 1 March 2019
}

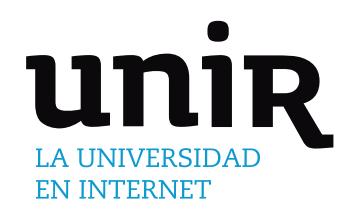

KEYWORDS

\author{
Artificial Neural \\ Network, Geographic \\ Information System, \\ Urban Development \\ Models, Soft Computing, \\ Fuzzy Logic, Spatial \\ Information Systems.
}

Exploring computational intelligence, geographic information systems and statistical information, a creative and artificial neural network as forecasting tools for describing the urban growth. This dynamic urban evolution and development. Then, these fuzzy clusters were modeled by the multi-layer neural networks to estimate the urban growth. Based on this novel intelligent model, the current state of development of Tehran's population and the future of this urban evolution were evaluated by empirical data and the achieved outcomes were detailed in qualitative charts. The input data-set includes four censuses with five-year intervals. Tehran's demographic evolution model forecasts the next five years with an overall accuracy of $81 \%$ and Cohen's kappa coefficient up to $74 \%$ beside the qualitative charts. These performance indicators are higher than the previous advanced models. The primary objective of this proposed model is to aid planners and decision makers to predict the development trend of urban population.

\section{INTRODUCTION}

$\mathrm{T}$ HE population growth and the migration of villagers to urban areas change the urban land use rapidly [1] and this paper models this urban evolution. According to United Nations (UN) reports, until 2030, about $60 \%$ of the world's population will be living in cities, and the average age of residents in Tehran, the metropolis chosen as a case study of this manuscript, would be 40 years old and the urban area in that year will be 1.2 billion square kilometers. Consequently, the spatial patterns of its urban areas will change dramatically by their boundaries. Because of the permanent changes in their shape and structure, modeling the evolution of urban areas is one of the most complex issues of research [2].

The decision-making process in urban environments requires a direct participation of a wide range of various aspects and a high level coordination among them. To have this process, governments typically conduct censuses. Demographic data is provided or reviewed at long intervals, such as five or ten years. In this range, the population will be assessed through non-qualitative and approximate estimations like the Average Migration Rate (AMR) or even more accurate statistical data such as Birth Rate (BR). Since the results of demographic estimates are chiefly inaccurate, and one cannot use them for providing an estimation of population dispersion, it is necessary to offer a model for appraising urban evolutions which also affect the decision-making process [3].

Typically, multi-centered contemporary cities, unlike old singlecentered towns, lead to the complex evolution model. Changes in

* Corresponding author.

E-mail address: mo_borhani@sbu.ac.ir urban dispersion and land use in metropolitan areas such as Tehran demand traffic planning and high-quality life and environment, therefore, due to issues such as the increased density and lack of useful space, this requirement is becoming more apparent every day [4]. The non-managed urban development processes like for example the expansion of transportation infrastructure, industrial structures, and other construction areas affect the environment and lead it to an unsustainable development [5], and once again an urban evolution model is needed here.

Modeling and simulating complex dynamic systems like urban areas could be done by using geographical data as well as computational intelligence techniques such as fuzzy logic and useful Artificial Neural Networks (ANNs). Computational intelligence algorithms are perfectly capable of determining the pattern of changes in land and land use in non-parametric methods and places with a high degree of heterogeneity [4]. In this paper, through fuzzy boundaries [6] a method for displaying spatial categories has been offered. This fuzzy clustering provides a better profile for any spatial unit. Moreover, neural networks [7] were used for forecasting complex nonlinear issues regarding demographic evolution. Neural networks are stable against noise, and they are quite capable of automation. Also, they have no assumption about the nature of the data distribution [8]. In this study, computational intelligence techniques were used for modeling urban growth and development [7] and also by assessing the urban population dispersion through spatial data systems as well as satellite images [9], joint time-location analysis and the prediction model of urban development trend were achieved. Finally, a method for estimating urban future through neural networks, fuzzy clustering, and geographical data systems was proposed.

This proposed model has been applied and implemented specifically for Tehran city. At first, fuzzy clustering was used for the 
classification of spatial units. These categories are introduced based on the statistical data of regions in Tehran, residential statistics, and their business information. By fuzzy clustering, each group is marked as an urban evolution parameter. Based on time series data, the neural network will be used for predicting the future of the city for any spatial unit.

This paper is organized as follows: in section II, diagrams of demographic growth and computational intelligence models that focus specifically on neural networks are presented. In section III, the proposed urban evolution model is described in detail and its innovations, as well as the causes of their impacts, are explicitly stated. In section IV, the case study of Tehran Metropolis using the real data achieved from censuses in four of the past five-year periods are introduced. In section $\mathrm{V}$, the results of this case study are demonstrated as qualitative maps and quantitative evaluators. Then the article ends with a conclusion in section VI and references.

\section{URBAN EVOLUTION AND COMPUTATIONAL INTELLIGENCE} Models

Urban growth is a complicated process, and so far different models have been introduced regarding the urban analysis and for studying the changes in the urban environment through physical, economic, and social parameters [10]. Also, many parameters with different behavioral patterns in different time-location scales have been investigated in these models [7]. From a basic look and due to differences in the definition of the city, various theories respecting the analysis and simulation of urban evolution have been offered. Also, some mathematical equations have been created for the simulation of the urban phenomenon [11]; however, the relation between these theories and models are usually weak and computational capabilities have not been used as a useful tool in these models [1]. Urban growth models are an interdisciplinary process which employs different technologies such as spatial data systems, remote sensing, urban geography, and complex theories at the same time. Concerning this city and its dynamics, geographical information systems and remote sensing are considered powerful tools for managing spatial data and extracting valuable knowledge [12] [14]. Baseline models [15] - [17], fractal models [18], and decision trees are also being used for modeling urban growth [19]. In the work done by Triantakonstantis, computational intelligence systems - as traditional models for analyzing spatial data - were employed for modeling the urban growth [19]. Regarding the land use, Omrani et al. used neural networks which were dynamic and had a discrete time - location [20]. Leo and Lodrope employed neural networks to detect new urban regions through the images of satellite sensors [21]. Their results indicated the value of neural networks in recognizing the changes. By using geographical information systems as well as artificial neural networks, Pichanoski et al. created a model for determining the land transformation based on social, political, and environmental parameters which are able of predicting the urban changes [22]. Moreover, Pichanoski et al. introduced an urban evolution model based on neural networks, geographical information system, and remote sensing. They employed a multilayered perceptron neural network, satellite images taken from 1988 and 2000, as well as social, economic, and environmental variables to estimate the expansion of metropolitan areas [23]. The combination of neural networks and fuzzy logic with experimental, conditional, and statistical methods lead to better results. For example, Guan and Clark used a dependent cellular automation model based on neural network backpropagation algorithm to simulate and forecast Beijing's evolution [24]. Dependent upon socio-economic data, this model could predict the future requests for urban space.

The previous studies suggest that in most cases, computational intelligence systems provide better natural and quantitative results than statistical methods. It is because the uncertainty principle is taken into account in this kind of methods. In most of these studies, the satellite images of remote sensing have been used and artificial neural networks, as well as a cellular automation model, were employed for simulating the urban evolution phenomenon. Regarding the neural network, time series data, satellite images, and some of the social and economic data were stored in spatial data systems and then neural networks were employed for predicting the urban evolution.

There are two main differences between the proposed method and previous studies. First of all, instead of using cellular raster data like the cellular automation model, we used statistical census analyses based on the descriptions of the urban situation, topographic information, fault, the city's green space, the distance of regions from main roads, and the distance from downtown in each spatial unit. Afterwards, we employed artificial neural networks to predict the future condition of the city. In the previous work, in the pixel based methods, the effects of other regions cannot be determined in a region for population growth in this region. The second distinction between this model and the works of the past is the usage of possibility theory and the correlation between data. In fact, in our proposed model, each region has not been investigated in separate pixels. They have been dealt with as fuzzy clustered areas with spatial information. The real changes in this paper have been studies based on time and the neighbors of each sector.

\section{The Proposed Urban Evolution Model Based on Soft COMPUTING}

In this section, simultaneous analysis of the spatial and temporal data based on computational intelligence and spatial data systems for modeling urban evolution is offered. This new method has been designed based on clustering areas of study and then artificial neural networks. The structure of the proposed method for urban growth model is depicted in Fig. 1.

In this method, spatial entities will be grouped by attributes. These attributes are population, population density, land use, building usage, proximity to downtown, distance from highways, distance from commercial areas, the slope of the region, and fault map.

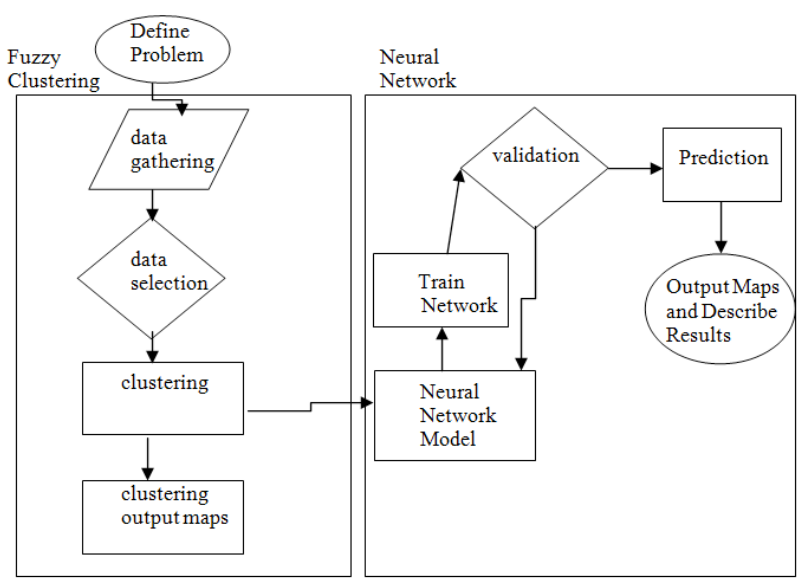

Fig. 1. The structure of the proposed method for urban growth model.

At first, the spatial reference was determined and then census and statistical data were collected. The census data at least covers four fiveyear periods. It is essential to select and discriminate these variables for the clustering process. This section is related to the available data. High correlated variables should be excluded from the calculations.

Clustering is a method used for recognizing the structure of data and fuzzy clustering as a proper spatial analysis is being used in 
different practices. The fuzzy C-Means clustering algorithm is one the most popular clustering algorithms [6], which have also been utilized in this study. In fuzzy C-Means algorithm, data is assigned to specific categories based on similarity and proximity.

In clustering algorithms - such as K-Means - each data is allocated to only one category, however, in fuzzy clustering, each data with a degree of membership is assigned to different groups. This membership grade for each class is a number between 0 and 1 . The closer the features of data to a particular category, the closer this number to 1 [25]. Wijayanto et al. used fuzzy clustering for modeling based on the mass of population [26]. Dimartinio et al. employed fuzzy clustering to introduce a fuzzy index to forecast spatial-temporal features [27]. In fuzzy clustering, every category has a center that indicates an object from that class. It is beneficial to use fuzzy clustering because these objects can be assigned to several categories with different membership degrees. On the contrary, in standard clustering, every object belongs to only one class. This indicates the level of membership value; i.e., how close the member is to the center of the categories. If the membership degree of an object relative to a class is $95 \%$, it means that object belongs to that class; on the other hand, if the membership degree is $5 \%$, it means that object has the least likeness to that class.

The algorithm of fuzzy clustering is like the classic average algorithm. In This method, the number of clusters is defined. The goal function of this algorithm is described as follows:

$$
J=\sum_{i=1}^{c} \sum_{k=1}^{n} u_{i k}^{m} d_{i k}^{2}=\sum_{i=1}^{c} \sum_{k=1}^{n} u_{i k}^{m}\left\|x_{k}-v_{i}\right\|^{2}
$$

In this function, $\mathrm{m}$ is a real number that is greater than one. In most cases $m$ is $2 . X_{k}$ is the $k_{t h}$ sample and $V_{i}$ is the center of cluster $i . U_{i k}$ defines the membership degree of sample i relative to the class $\mathrm{k}$. the sign $\|*\|$ is the degree of samples to a center of cluster. With values $u_{i k}$, a matrix can be defined, that has $\mathrm{c}$ rows and $\mathrm{n}$ columns. The numbers in this matrix are between 0 and 1 , but the sum of the numbers in each column is 1:

$$
\sum_{i=1}^{c} u_{i k}=1, \forall k=1, \ldots, n
$$

Using the above condition and minimizing the goal function, the center of cluster and the membership degree of sample i relative to the class $\mathrm{k}$ are computed by below functions:

$$
\begin{gathered}
u_{i k}=\frac{1}{\sum_{j=1}^{c}\left(\frac{d_{i k}}{d_{j k}}\right)^{2 /(m-1)}} \\
v_{i}=\frac{\sum_{k=1}^{n} u_{i k}^{m} x_{k}}{\sum_{k=1}^{n} u_{i k}^{m}}
\end{gathered}
$$

The steps of this algorithm are described as follows:

- Define the first value for $\mathrm{c}, \mathrm{m}$

- Calculate the center of clusters

- Calculate the membership to each class

- If $\|\mathrm{Ul}+1-\mathrm{Ul}\| \leq \varepsilon$, then the algorithm is finished, else come back to step 2 [28].

In this paper, fuzzy clustering through C-Means algorithm was used for creating the categories which determine both the urban degree for each region and a particular grade of urban growth. These classifications show the evolution of an area in a populated urban area. In any spatial reference, the membership degree for each class for every five years will be determined. As a result, the urban condition of each spatial unit will be determined based upon that particular category.

Changes in the membership degree of spatial units that occur in different decades may change their categories. For instance, a region might belong to a category with a meager population growth whose membership degree over a 5 -year period is $58 \%$ but in the next 5 -year period, the membership level of this particular region would be $52 \%$, and it would belong to a category with a small population growth. Changes in membership degree might happen in regions that their categories never alter. For example, an area belongs to a minimal demographic group whose membership degree is $88 \%$ over the first five years, and then it becomes $65 \%$ in the next five years, and lastly, it turns to $52 \%$ in the last five years. Therefore, this region is always in a category with a tiny population. Fuzzy clustering can demonstrate the decrease in membership degrees in these decades, changing from an area with a very low population density into another category. As a result, we will be able to get a better perception of the public profiles of a region, and then we can easily describe these predicted changes.

It is because these existing entities can be placed in different categories and their changes become evident over time. Once the process is done, the results will be recorded in a database, and then they will be displayed through spatial data systems, and we will be able to track the trend of each category [29]. Afterwards, this proposed structure will forecast and study the urban evolution - right after the fuzzy clustering of the place is determined. The prediction of urban growth in each spatial unit (cluster) will be made through neural networks with time series data. In the proposed structure, time series data will be improved by using spatial relations. Each unit shares borders with other units, and this identifies the same relationships between the characteristics of the groups.

It is expected that these changes in adjacent areas in a region affect another area. As a result, it is impossible to predict the growth state of a zone solely based on time series data. Therefore, in this paper, the urban evolution was identified based on the correlations between one region and its adjacent areas. Spatial data systems were used for determining the border of each region with its neighbors and also for measuring the boundary length. In this method, the new percentage value for each area is determined based on these boundaries. For instance, a region belongs to a tiny population in the neighborhood of the border, $32 \%$ is related to a small population, $45 \%$ belongs to a small population, and $23 \%$ belongs to an average population. Although the boundary length is not the only parameter that is needed to be identified for spatial interference, we just used this setting for simplification.

Time series data for each spatial unit and the adjacent units will be put into the input tables of neural networks. In this method, the neural network will be trained for learning the changes in a region and also identifying the impact of adjacent areas on that region. This structure uses these inputs, the history of spatial units, and their neighbors to predict the condition of that area in the future. A set of parameters for each neural network must be set.

Artificial neural network is a non-linear model that acts similar to a human neural system. Each ANN consists of a series of nodes and weighted connections between them [30].

A schematic diagram of the structure of an ANN is shown in Fig. 2 [30]. Each node in an ANN, receives an input from neighboring node, performs some calculation and sends the results to the other nodes as an output

In fact nodes are connected by weighted connectors to different 
layers in the ANN. In ANN there are different layers that can be generally divided into three groups of input layers, hiding layers and output layers [30].

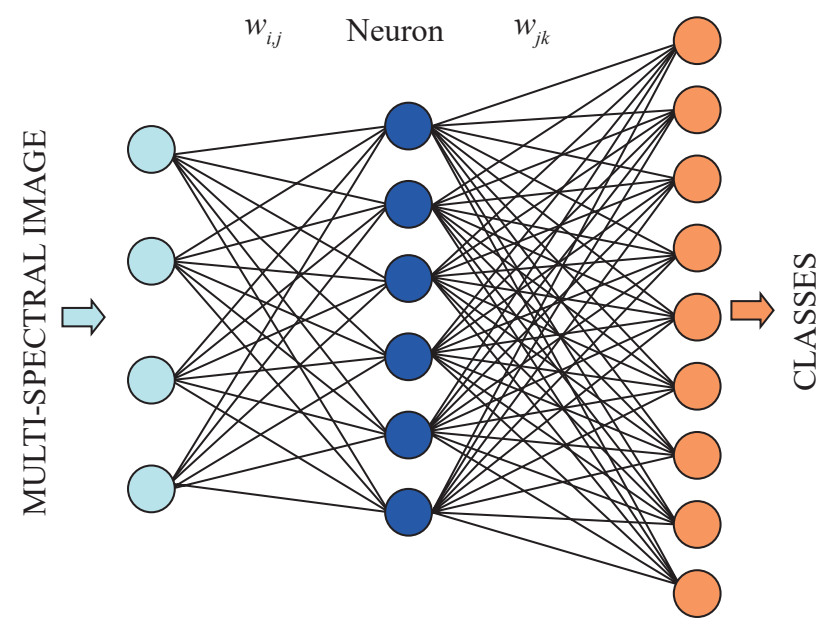

Inlet level Hidden level Outlet level

Fig. 2. Schematic diagram of the structure of an ANN [30].

ANN consists of two stages in classification, training and recalling [31]. In the training stage, an operation is being conducted to improve the connection weighting coefficients through some iteration processes. In this training stage the known inputs and outputs are used, and consequently is called Back-Propagation Supervised Classification [33]. This method is a gradient iteration algorithm that is being used to minimize the error as is shown in equation 5.

$$
E=\frac{1}{2} \sum_{j=1}^{L}\left(d_{j}-o_{j}^{M}\right)^{2}
$$

where, $d_{j}$ and $o_{j}^{M}$ are the desired output and ANN output respectively. Based on the results of equation, the amount of correction for each weight can be calculated [32].

$$
\left\{\begin{array}{l}
\Delta w_{i, j}=-\eta \frac{\partial E}{\partial w_{i, j}} \\
\Delta w_{i, j}(t+1)=\Delta w_{i, j}+\alpha \Delta w_{i, j}(t)
\end{array}\right.
$$

Here $\mathrm{W}$ is the weight between two nodes and $\eta$ is a positive constant parameter that is being used for adjustment and is called training rate. $\alpha$ is a momentum factor (usually called stability factor) and can take a value between 0 and 1 and $t$ is the number of iterations [32]. In the recalling stage, ANN works according to the trained weighting coefficients, and conducts interpolations and extrapolations. These parameters include the number of intermediate layers, the number of neurons in each layer, training rates, momentum, the number of repetitions, and activity functions. It is important to adjust each parameter correctly because the ultimate accuracy and the acceptance of any neural network depends on this action. One of the privileges of ANN method compared to traditional statistical methods is that the networks are free in distribution i.e. the training and recalling are depending on the linear combination between data patterns and are independent of input data [30]. However the reasons for the success of ANN in classification can be summarized as: 1) there is no need for pre-assumption in data distribution 2) it is faster compared to the other methods 3 ) it permits user to make use of the initial knowledge regarding classes and possible limitations 4 ) the method is able to manage the spatial data from multi-sources and can achieve their classification results equally [30].

Concerning neural networks, user experiences and right and wrong actions are considered necessary elements. Afterwards, some statistical indicators such as least squares error and correlation coefficient will be evaluated, and their acceptance rate will be identified. Predictive maps will be designed in the next step. In other words, the outputs of the neural network will be close to the reference outputs.

\section{The Case Study of the Proposed Model Regarding TEHRAN METROPOLIS}

The proposed model has been implemented for modeling the urban evolution of Tehran Metropolis in all 22 zones of the municipality. Tehran's regions include small areas which are themselves like a city. The capital of Iran, Tehran, has been chosen as a case study because it possesses some particular features such as the high focus of industry in the city, manufacturing activities, services, and workforce. The area of Tehran is about $730 \mathrm{~km}^{2}$, and its population is about 8 million, which is divided into 22 local zones. Fig. 3 demonstrates Tehran's geographical location.

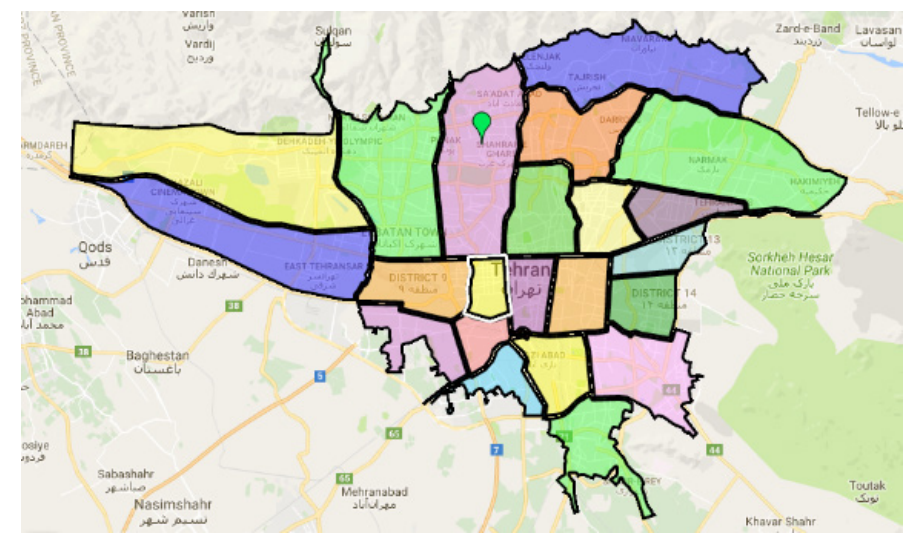

Fig. 3. Case study, Tehran.

\section{A. Data}

Due to the existence of control information, the spatial unit reference in this research was considered the municipalities. The data of Iran's statistical services centers include building usage and the population from 1996 to 2011. Statistical information relative to 2001 was collected through interpolation. Census variables are being used for defining the "Urban Evolution Model," while it is possible that other variables will be studied in future articles.

The data include four consecutive five-year censuses, and each census comprises eight parameters as follows: 1. Population, 2. The number of residential usages, 3. Distance from downtown, 4. Distance from fault, 5. Distance from highways, 6. Green space, 7. Industrial practices, and 8. The slope map. Some of them are shown in Fig. 4. Through these variables and by population, population growth rate, and building usages, the profile of municipalities that are well suited to the public profile will be acquired. For instance, the high number of buildings and a significant population indicate the downtown.

\section{B. The Results of the Implementation of Clustering Analysis}

The fuzzy C-Means algorithm was used for the required clusters. Clustering was done for every five years, and the categories suggest the urban population grade for each region. Five categories named very low population, low population, average population, high population, and excessive population were considered. 


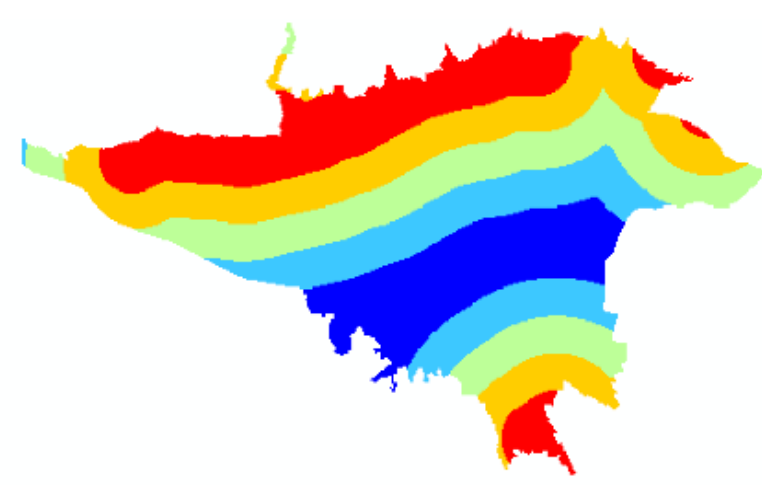

(a)

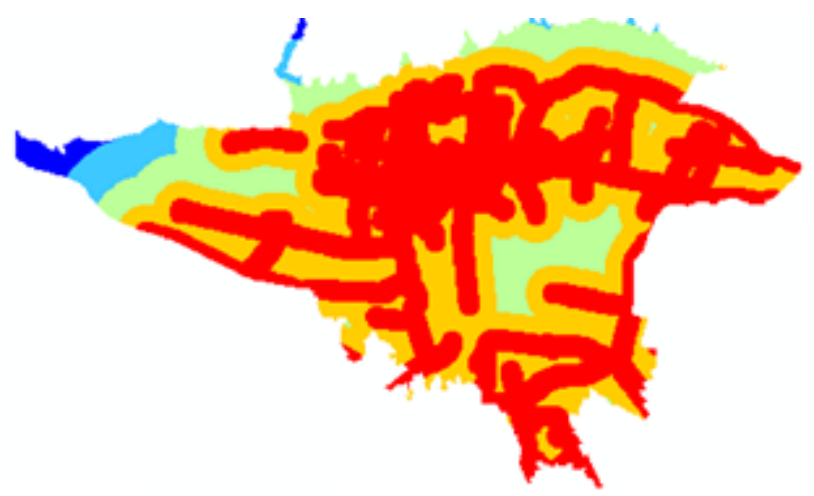

(b)

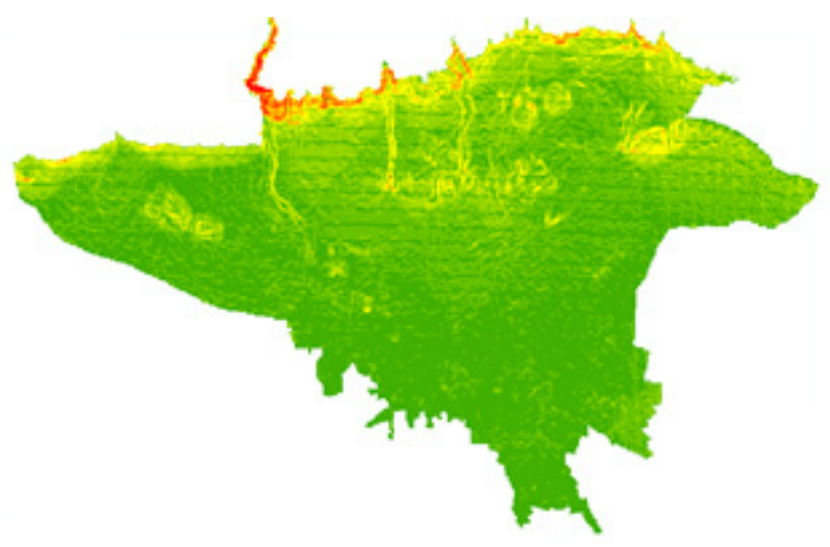

(c)

Fig. 4. (a) Distance from fault, (b) distance from main roads, (c) Tehran's slope maps A-C.

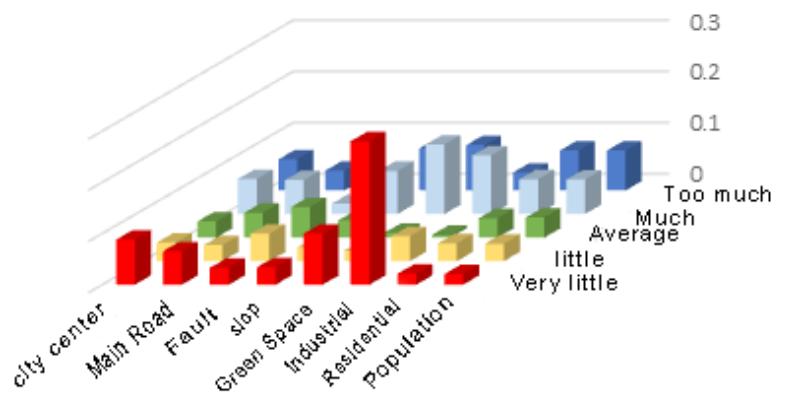

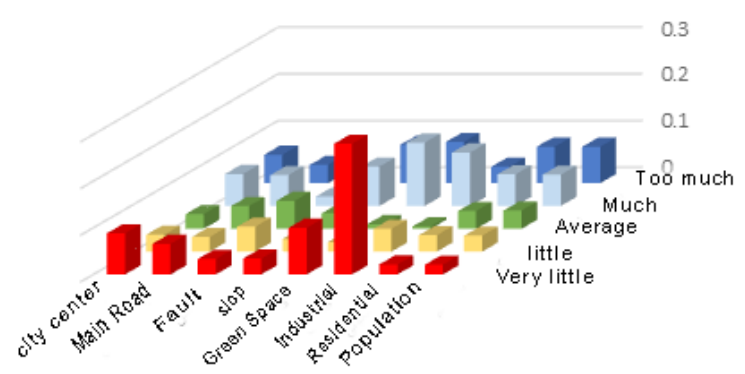

(b)

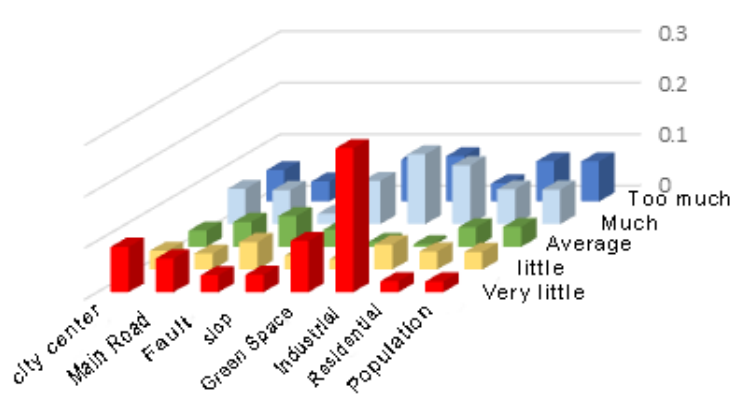

(c)

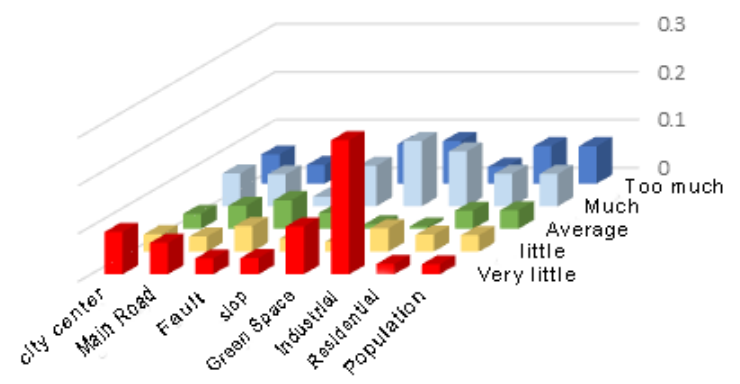

(d)

Fig. 5. The category centers in four five-year periods: (a) 1996, (b) 2001, (c) 2006, and (d) 2011.

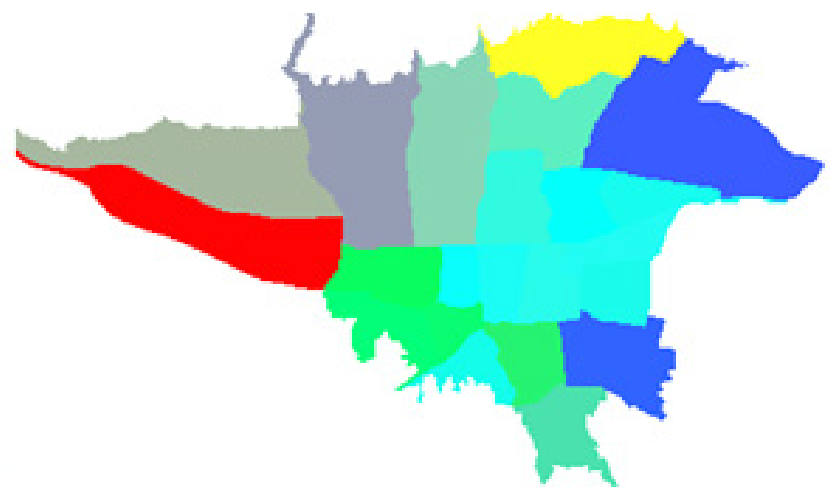

(a)

(a) 


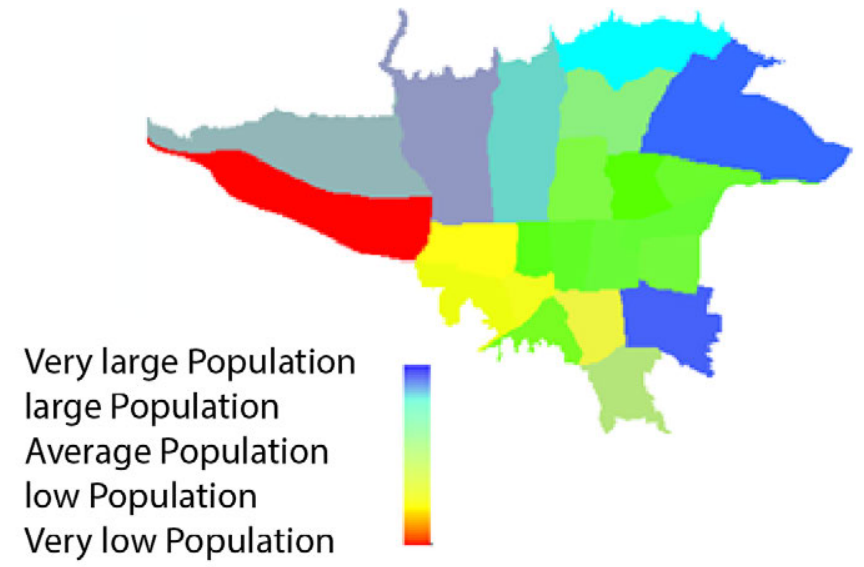

(b)

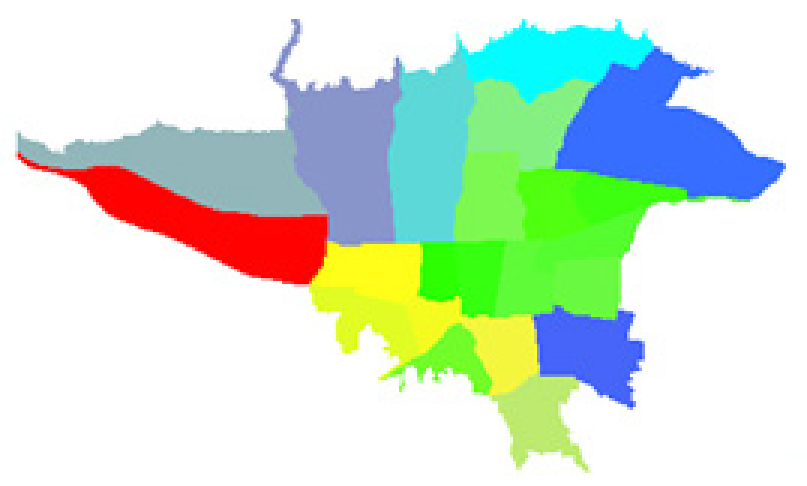

(c)

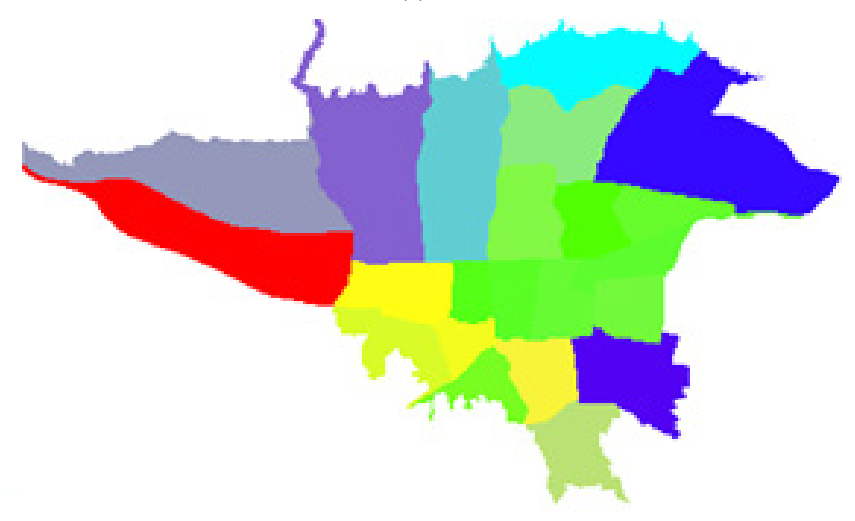

(d)

Fig. 6. Clustering Tehran's regions using C-Means algorithm in (a) 1996, (b) 2001, (c) 2006, and (d) 2011.

Fig. 5 indicates the centers of the categories over the four 5-year periods - 1996, 2001, 2006, \& 2011 - and each class were described based on the values of the group centers. As you can see, those categories with a very small population have the most industrial activities, and the highest amount of green space is perceived in the categories with a large population. Each region belongs to classes with different grades over five years; e.g., if an area belongs to the category with a very low population in 2011, its statistical values are similar to the category with a very low population. Hence, the centers of categories refer to the statistical values in every five years. Each group has been described based on its center as follows:

- Very low population: in these areas, compared to the other sectors, a low amount of people resides. These areas are far from the downtown and the main roads. There are many factories in these regions which influence its weather conditions.

- Low population: these areas have a low population and few green spaces. They are close to the downtown. Most of the offices are located there. They are almost close to the main roads.

- Average population: this category has an average population. There are few industrial regions there. These areas are near the downtown and main roads.

- High population: these areas have a quite excessive population. They are far from the downtown, and they have many green spaces.

- Very high population: these areas have many green spaces and are densely populated. Many buildings can be seen in these regions. They have few industrial zones, and they are close to the main roads.

In every five years, each region from membership degree of fuzzy C-Means algorithm will belong to one of these classes. Fig. 6 shows the clustering of these membership degrees over the four five-year periods - 1996, 2001, 2006, \& 2011.

Through this clustering, we will be able to understand each spatial unit with its membership degree changes better while other clustering methods are unable to track changes. In these categories, Fuzzy Logic of the urban population for each class is determined. Through this process, the trends of each region will be identified, and then the city's future urban evolution will be simulated. For example, in Fig. 6, the increase in urban population in Tehran's northern and western regions is presented. Due to Tehran's air pollution in central areas, its residents would rather live in northern regions which have a more suitable climate. This is entirely evident in Tehran's northwest regions. For instance, $35 \%$ of the residents in Tehran's zone 5 preferred populated areas in 1996 while in 2011, with a 46-percent membership degree, it was named a populated zone. These changes are visible in Fig. 7; the color of this district changes from red (low population) to blue (high population). The northeastern regions of Tehran have a better climate; hence, many people have been living there during these years. On the other hand, the population of Tehran's central areas was decreasing, and people preferred to live in a field with average population.

\section{The Implementation of Neural Networks for Prediction}

Regarding the prediction of the city's growth, the multilayer perceptron neural network was chosen and implemented. The implementation of input data has ten features of which the first 5 data include the membership degree of each region for every five years. Data sixth to tenth contain the average degree of membership of the surrounding areas for the same five years. The outputs of this model comprise 5 data that indicate the membership degree in the next five years. Consequently, the future state of the city development will be determined. $70 \%$ and $30 \%$ of the data were selected for training and testing respectively. To reach a proper network, more than 50 combinations of neural network settings were employed for trial and error. This suitable system has an intermediate layer relative to five neurons in a linear function. The amount of momentum training for two layers was considered 0.5 . Based on the results, the weights were selected with the highest accuracy. For example, minimum mean squared error for training data after 1000 repetitions was 0.0001 . Table I presents the architecture (i.e., the intermediate layers, conversion functions, step size, and momentum size) of the neural network which has been achieved after 1000 repetitions in the trial and error phase. Through test data, as follows, the neural network was tested. The test data were not used during training. The real data was employed to test the neural network. The reference output and system output for a part of the test data are presented in Fig. 8. The neural network was trained for determining the data, and then it got prepared for prediction. 
TABLE I. The Neural Network Settings

\begin{tabular}{|c|c|c|c|c|c|}
\hline $\begin{array}{c}\text { The } \\
\text { number } \\
\text { of hidden } \\
\text { layers }\end{array}$ & $\begin{array}{c}\text { The } \\
\text { number of } \\
\text { neurons in } \\
\text { layer 1 }\end{array}$ & $\begin{array}{c}\text { The } \\
\text { number of } \\
\text { neurons in } \\
\text { layer 2 }\end{array}$ & $\begin{array}{c}\text { The } \\
\text { number of } \\
\text { repetition }\end{array}$ & $\begin{array}{c}\text { Minimum } \\
\text { square } \\
\text { error }\end{array}$ & $\begin{array}{c}\text { Overall } \\
\text { accuracy } \\
\text { of test } \\
\text { data } \\
\text { (percent) }\end{array}$ \\
\hline 1 & 5 & 0 & 1000 & 0.0002 & $\mathbf{7 9}$ \\
\hline 1 & 10 & 0 & 737 & 0.0001 & $\mathbf{8 0}$ \\
\hline 2 & 10 & 10 & 507 & 0.0001 & $\mathbf{8 0}$ \\
\hline 2 & 20 & 20 & 239 & 0.0001 & $\mathbf{4 6}$ \\
\hline
\end{tabular}

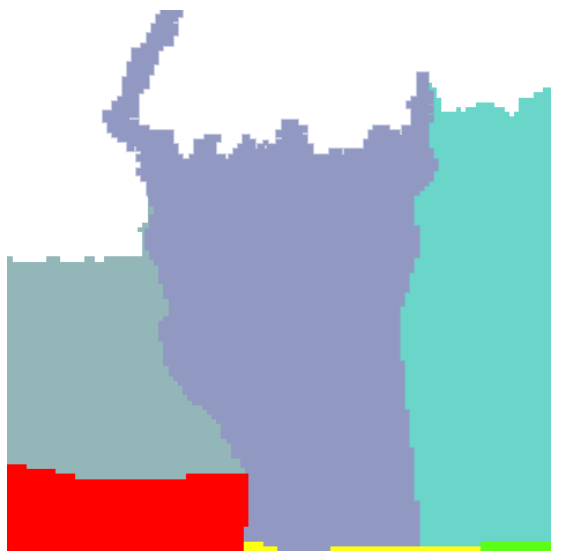

(a)

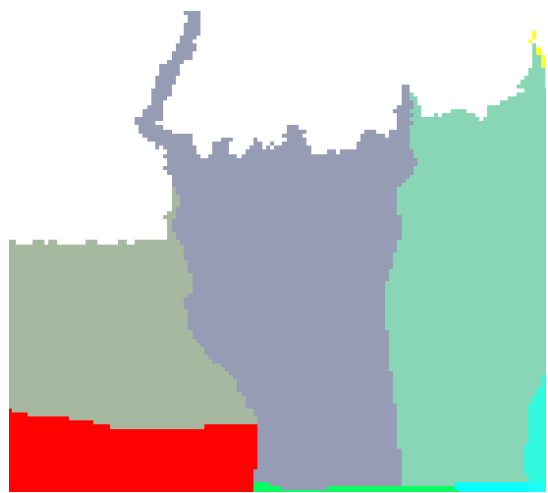

(b)

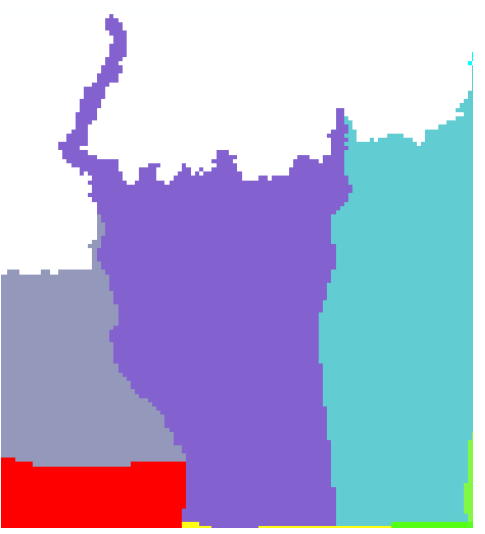

(c)

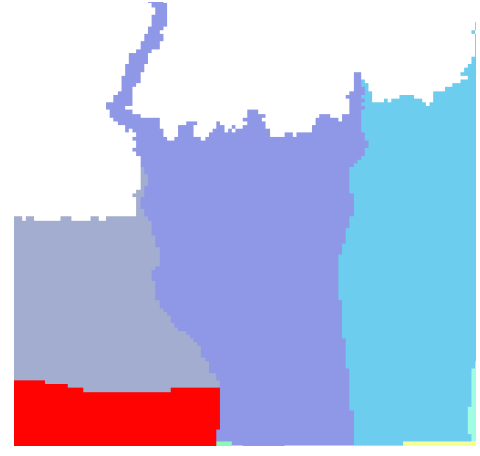

(d)

Fig. 7. The membership degree changes in Tehran's district 5 in (a) 1996, (b) 2001, (c) 2006, and (d) 2011.

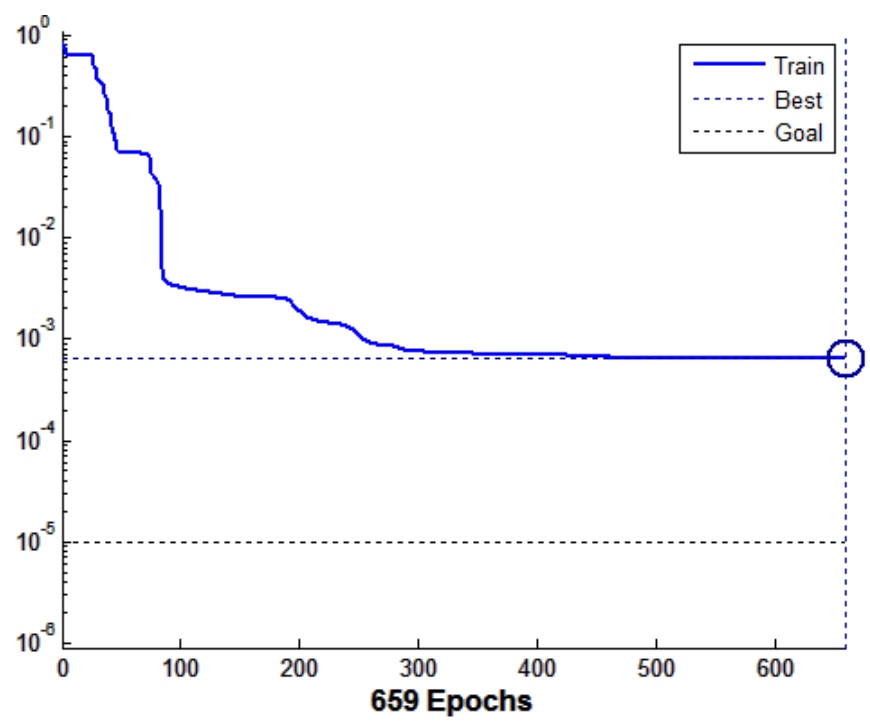

Fig. 8. The minimum square error of artificial neural network training.

\section{Evaluation of Results and Discussion of Prediction}

The confusion matrix was used for evaluating the quantity results gained from the prediction of the neural network. This matrix arises from data to data and known data to corresponding data comparisons of the classification results. In this matrix, the known data are represented in columns, and the data of classification results are shown in rows. Two critical parameters named overall accuracy and Kappa coefficient were used for evaluation. The overall accuracy is the average of classification accuracy that considers the proportion of data with a correct classification of the total data. In fact, by removing the effect of chance on classification, the amount of compliance with the actual data will be specified. These results can be seen in Table II.

TABLE II. USING THE CONFUSION MATRIX TO DETERMINE THE ACCURACY OF PREDICTION

\begin{tabular}{ccccccc} 
Category & $\begin{array}{c}\text { Very } \\
\text { low }\end{array}$ & Low & Average & High & $\begin{array}{c}\text { Very } \\
\text { high }\end{array}$ & Total \\
\hline Very low & 2 & 1 & 0 & 0 & 0 & 3 \\
Low & 0 & 8 & 0 & 1 & 0 & 9 \\
Average & 0 & 0 & 13 & 0 & 0 & 13 \\
High & 0 & 0 & 0 & 2 & 2 & 4 \\
Very high & 0 & 0 & 0 & 3 & 5 & 8 \\
Total & 2 & 9 & 13 & 6 & 7 & 37
\end{tabular}


The data collected from 2011 was used for predicting 2016. The outcomes of the membership degrees were related to the regions, and the results are presented in Fig. 9.

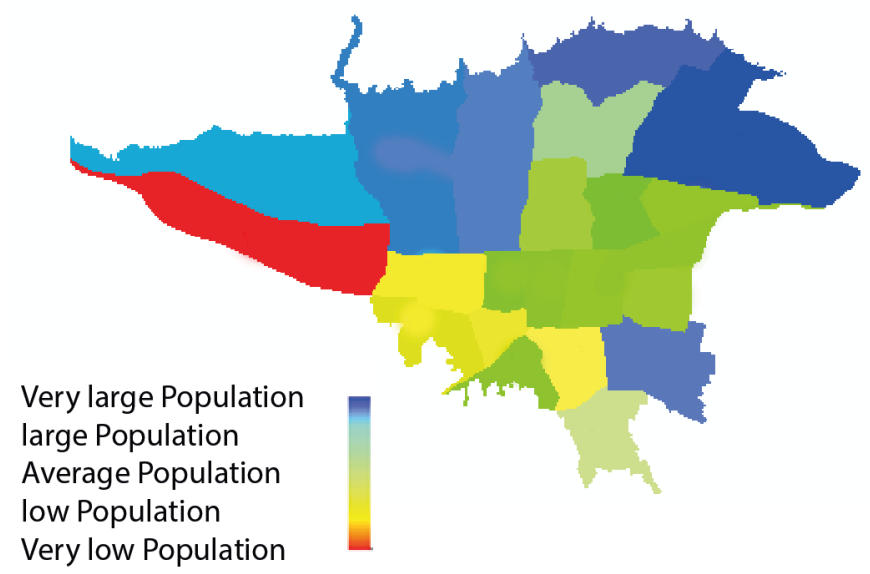

Fig. 9. Neural network prediction of population growth procedure in 2016.

\section{E. Discussion}

In this proposed method for modeling the urban evolution, fuzzy clustering and neural networks were used for analyzing and predicting the urban growth. Fuzzy clustering offers some descriptions of the urban condition of any spatial unit, and then a neural network will forecast the future state of the city. Building and population data were employed for estimating the urban growth; however, satellite images were never used.

A densely populated area could be considered a place for recruiting residents and constructing new buildings. As a result, the urban growth distribution will be specified. Through the predictive maps (Fig. 9), as you can see, Tehran's northern and northwestern area is populated, and as it is predicted, its central areas will become average or low populated areas. According to the predictions, zone 5 will have a high population, and it will require a proper planning for resource allocation.

Population growth in northern and northwestern areas of Tehran causes more traffic and effects on air pollution. In addition, population density raises the needs for resources.

According to the results of the proposed method, the reason of low population in south and southwestern areas should be taken under consideration and try to fix the defects in order to have most equally distributed population all over the city.

The proposed method has some limitations such as resolution power in the units of these areas. In the future, these studies can be useful for areas inside each region. Moreover, by using remote sensing data, we will be able to improve the capabilities of the algorithm.

\section{CONCLUSION}

Urban evolution can be modeled through fuzzy clustering and neural networks. Although the proposed method is simple, it can be adapted to instantaneous dynamic structures. Fuzzy clustering is an appropriate means and was used in this model. Consequently, each spatial unit has its profile over every five years. Fuzzy Logic was used to create a full picture of the areas studied for five years and also it presented the membership degree changes and upcoming trends for each spatial unit.

Through the neural network, the future urban state of each region was specified and, as a result, a better profile for decision-making was offered. In the future, this method with more temporal data and a completed subject database could become an efficient spatial model. Practical methods are precious regarding Tehran City because they determine the tendencies of areas and development of buildings in the future.

\section{REFERENCES}

[1] E. Vaz, P. Nijkamp, "Gravitational forces in the spatial impacts of urban sprawl: An investigation of the region of Veneto, Italy", Habitat International, 2015.

[2] L. Jiao "Urban land density function: A new method to characterize urban expansion", Landscape and Urban Planning: 26-39, 2015.

[3] R. Aghataher, N. N.Samani, "Spatial Demographic Distribution Zoning of a City with Area Interpolation Method using Image-based Geo-spatial Information System-Case Study: Tehran City", Journal of Geospatial Information Technology, Iran (4): 65-82, 2016.

[4] J. Wang, G. Mountrakis "Developing a multi-network urbanization (MuNU) model: A case study of urban growth in Denver", International Journal of Geographical Information Science, 25(2): 229-253, 2011.

[5] J. Barredo, C. Lavalle, M. Kassanko,"Urban Scenario Modeling and Forecast for Sustainable Urban and Regional Planning, in GIS for sustainable development. M. Campagna, Eds., CrcPres", Taylor \& Francis: 329-349, 2005.

[6] G. Grekousis, T. Hatzicheros, "Comparison of two fuzzy algorithms in geodemographic segmentation analysis" The fuzzy C-Means and Gustafson Kessel methods, Applied Geography (34): 125-136, 2012.

[7] J. Cheng, "Modeling spatial and temporal urban growth", PhD Dissertation, Utrecht University, 2003.

[8] J. D. Olden, D. A. Jackson, "Fish-habitat relationships in lakes: Gaining predictive and explanatory insight by using artificial neural networks", Transactions of the American Fisheries Society (130): 878-897, 2001.

[9] S. Mohammady, M. R. Delavar, "Urban sprawl assessment and modeling using LANDSAT images and GIS" Modeling Earth Systems and Environment 2.3: 155, 2016.

[10] O. Hamdy, S. Zhao, T. Osman, M. A.Saleheen, Y.Eid, “Applying a Hybrid Model of Markov Chain and Logistic Regression to Identify Future Urban Sprawl in Abouelreesh", Aswan: A Case Study, Geosciences, 6(4): 43, 2016.

[11] G. Grekousis, M. Panos, N. Yorgos, "Modeling urban evolution using neural networks, fuzzy logic and GIS, The case of the Athens metropolitan area", CITIES (30):193-203, 2013.

[12] S. Movahedi, M. Taleai, M. Karimi, "Development of a GIS-based model for Locating Urban Neighbourhood and District Centres based on Mixed Land Use Concepts", Journal of Geospatial Information Technology,Vol.3, No.3, autumn, 2015.

[13] J. Kubanek, E. Nolte, M. H. Taubenbock, F. Wenzel, M.Kappas," Capacities of remote sensing for population estimation in urban areas", Journal of Earthquake Hazard Impact and Urban Planning Environmental Hazards: 45-66, 2014.

[14] J. Kumar, P. K. Garg, D. Khare, "Monitoring and modeling of urbansprawl using remote sensing and GIS techniques", International Journal of Applied Earth Observation and Geoinformation, 10(1): 26-43, 2008.

[15] I. Benenson,"Multi-agent simulations of residential dynamics in the city, Computers", Environment and Urban Systems, 22(1): 25-42, 1998.

[16] J. Kerridgw, J. Hine, M. Wigan, "Agent-based modeling of pedestrian movements: The questions that need to be asked and answered", Environment and Planning B: Planning and Design (28): 327-34, 2001.

[17] F. Hosseiali, M. Azizkhani, "Developing an agent-based model for spatial simulation of pedestrian's behavior passing across the street and using the pedestrian bridges", Journal of Geospatial Information Technology, Volume 4, Number 2 (9-2016), 2016.

[18] G. Shen, "Fractal dimension and fractal growth of urbanized areas", International Journal of Geographical Information Science, 16(5):419437, 2002.

[19] D. Triantakonstantis, G. Mountrakis, J. Wang, "A spatially heterogeneous expert based (SHEB) urban growth model using model regionalization", Journal of Geographic Information System(5): 195-210, 2011.

[20] H. Omrani, A. Tayyebi, B. Pijanowski, "Integrating the multi-label landuse concept and cellular automata with the artificial neural network-based Land Transformation Model: an integrated ML-CA-LTM modeling framework", 2016. 
[21] X. Liu, J. R. Lathrop, "Urban change detection based on artificial neural network", International Journal of Remote Sensing, 23(12):2513-2518, 2002.

[22] B. C. Pijanowski, D. G. Brown, B. A. Shellito, G. A. Manik, "Using neural networks and GIS to forecast land use changes: A land transformation model", Computers, Environment and Urban Systems(6): 553-575, 2002.

[23] B. C. Pijanowski, A. Tayyebi, M. R.Delavar, M. J. Yazdanpanah, “ Urban expansion simulation using geo-spatial information system and artificial neural networks", International Journal of Environmental Research,3(4):493-502, 2009.

[24] Q. L. W. Guan, K. C. Clarek, "An artificial-neural-network-based, constrained CA model for Simulating urban growth", Cartography and Geographic Information Science, 32(4): 369-380, 2005.

[25] G. S. Chandel, P. Kailash, S. Man, “A Result Evolution Approach for Web usage mining using Fuzzy C-Means Clustering Algorithm", International Journal of Computer Science and Network Security (IJCSNS) (16.1): 135, 2016.

[26] A. W. Wijayanto, A. L. Purwarianti, H. Son, "Fuzzy geographically weighted clustering using artificial bee colony: An efficient geodemographic analysis algorithm and applications to the analysis of crime behavior in population", Applied Intelligence: 377-398, 2016.

[27] F. Di Martino, R.Mele, S.Sesa, U.Barillari, M.Barillari, "WebGIS based on spatio-temporal hot spots: an application to oto-laryngo-pharyngeal diseases", Soft Computing, vol. 20, (6): 2135-2147, 2016.

[28] R. Hathaway, J.Bezdek, "Nerf C-Means: Non-Euclidean Relational Fuzzy clustering", Pattern Recognition, 27 , 429-437, 1994.

[29] M. Borhani and H. Ghassemian, "Kernel Multivariate Spectral-Spatial Analysis of Hyperspectral Data," in IEEE Journal of Selected Topics in Applied Earth Observations and Remote Sensing, vol. 8, no. 6, pp. 24182426, June 2015. doi: 10.1109/JSTARS.2015.2399936.

[30] F. Caravajal, E.Crisanto, F.Aqguera, M. A. Aguilar, "Greenhouse detection using neural network with a very high resolution satellite image.", ISPRS Technical Commission II Symposium, 37-42, 2006.

[31] M. Mokhtarzadeh, M. J.ValadanZoej, "Road detection from high resolution satellite images using artificial neural networks", Int. J. of Appl. Earth Observation and Geoinformation,32-40, 2007.

[32] G. Y. Yang, "Geological mapping from multi-source data using neural networks". M.Sc. Thesis. University of Calgary, Canada, 1995.

[33] M. Borhani, Corpus Analysis Using Relaxed Conjugate Gradient Neural Network Training Algorithm, Neural Processing Letters, 2018. https://doi. org/10.1007/s11063-018-9948-8

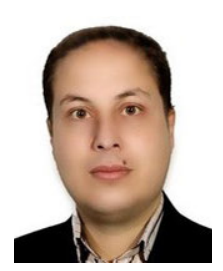

Mostafa Borhani

Mostafa Borhani received the BSEE degree and MSEE degree from Sharif University of Technology, in 2002 and 2004, respectively and he successfully defended his doctoral thesis entitled "Hyperspectral Remote Sensing Image Classification Based On Spectral-Spatial Kernel Methods" at the Department of Electrical and Computer Engineering, Tarbiat Modares University in 2015. His research interests include machine learning, artificial intelligence, kernel methods, adaptive algorithms and joint time-frequency analysis with special focus on Holy Quranic data analysis to achieve more comprehension and understanding on this holy book. His research is conducted as faculty member of the Quran Miracle Research Institute of the Shahid Beheshti University.

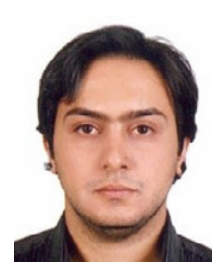

\section{Nima Ghassemloo}

Nima Ghassemlo received the BS degree from Tabriz University, in 2007 and MSEE degree in 2010, respectively and he is Ph.D. candidate at the GIS and remote Sensing Center of Shahid Beheshti University since 2016. 